\title{
Biomarkers of cardiovascular disease: contributions to risk prediction in individuals with diabetes
}

\author{
Katherine N. Bachmann ${ }^{1,2}$ • Thomas J. Wang ${ }^{1,3}$
}

Received: 19 April 2017 / Accepted: 10 July 2017 / Published online: 28 September 2017

(C) Springer-Verlag GmbH Germany 2017

\begin{abstract}
Cardiovascular disease is a leading cause of death, especially in individuals with diabetes mellitus, whose risk of morbidity and mortality due to cardiovascular disease is markedly increased compared with the general population. There has been growing interest in the identification of biomarkers of cardiovascular disease in people with diabetes. The present review focuses on the current and potential contributions of these biomarkers to predicting cardiovascular risk in individuals with diabetes. At present, certain biomarkers and biomarker combinations can lead to modest improvements in the prediction of cardiovascular disease in diabetes beyond traditional cardiovascular risk factors. Emerging technologies may enable the discovery of novel biomarkers and generate new information about known biomarkers (such as new combinations of biomarkers), which could lead to significant improvements in cardiovascular disease risk prediction. A critical question, however, is whether improvements in risk prediction will affect processes of care and decision making in clinical practice, as this will be required to achieve the ultimate goal of improving clinical outcomes in diabetes.
\end{abstract}

Electronic supplementary material The online version of this article (https://doi.org/10.1007/s00125-017-4442-9) contains a slideset of the figures for download, which is available to authorised users.

Katherine N. Bachmann

Katherine.n.bachmann@vanderbilt.edu

1 Vanderbilt Translational and Clinical Cardiovascular Research Center, Vanderbilt University School of Medicine, Nashville, TN, USA

2 Division of Diabetes, Endocrinology, and Metabolism, Vanderbilt University Medical Center, 2213 Garland Avenue, MRB IV Suite 7465, Nashville, TN 37232, USA

3 Division of Cardiovascular Medicine, Vanderbilt University Medical Center, Nashville, TN, USA
Keywords Cardiac complications - Clinical diabetes . Clinical science $\cdot$ Epidemiology $\cdot$ Human $\cdot$ Macrovascular disease $\cdot$ Review

$\begin{array}{ll}\text { Abbreviations } \\ \text { ACC } & \text { American College of Cardiology } \\ \text { AHA } & \text { American Heart Association } \\ \text { ESC } & \text { European Society of Cardiology } \\ \text { hsCRP } & \text { High-sensitivity C-reactive protein } \\ \text { hsTnT } & \text { High-sensitivity troponin T } \\ \text { NRI } & \text { Net reclassification improvement } \\ \text { NT-proBNP } & \begin{array}{l}\text { N-terminal prohormone of b-type } \\ \text { natriuretic peptide }\end{array} \\ \text { UKPDS } & \text { UK Prospective Diabetes Study }\end{array}$

Introduction

The elevated cardiovascular risk in individuals with diabetes mellitus has been recognised for decades [1-3] and has become an increasingly important public health concern in recent years given the dramatic growth in diabetes prevalence. Cardiovascular disease remains a leading cause of morbidity and mortality in individuals with diabetes. Thus, there has been increasing interest in identifying biomarkers of cardiovascular disease to allow better-targeted therapies for the prevention and treatment of cardiovascular disease in people with diabetes. There have been several review articles published on the topic of cardiovascular biomarkers in diabetes [4-6]. The present article focuses on the current and potential contributions of these biomarkers to cardiovascular disease risk prediction in individuals with diabetes. 
Current approaches to cardiovascular disease risk prediction in diabetes

Considers diabetes as a cardiovascular disease risk equivalent

1 Treats all individuals with diabetes as if they have cardiovascular disease

Assesses global risk of cardiovascular disease in individuals with diabetes using multivariable prediction models

2

Uses prediction models developed in the general population

Uses prediction models developed in populations with diabetes

\section{Current approaches to cardiovascular disease risk prediction in diabetes}

There are three prevailing approaches to cardiovascular disease risk assessment in diabetes (as summarised in the Text box). The first approach considers diabetes as a cardiovascular disease risk equivalent and treats all people with diabetes as if they have cardiovascular disease [7]. The second and third approaches both assess the global risk of cardiovascular disease in individuals with diabetes using multivariable prediction models. The second approach uses prediction models developed in the general population and applies them to people with diabetes [7]. Thus, the same model is used for individuals with and without diabetes. These multivariable models treat diabetes status as a risk factor, much like conventional cardiovascular disease risk factors such as smoking and age. The third approach uses prediction models developed in populations with diabetes, intended for use specifically in people with diabetes [7]. Implicit in this approach is the belief that separate models should be used for individuals with diabetes and for individuals without diabetes.

With regard to the first approach, diabetes has often been regarded as a cardiovascular disease risk equivalent because individuals with diabetes experience a markedly elevated incidence of cardiovascular disease [3]. However, classifying all individuals with diabetes as having a cardiovascular disease risk equivalent may be an oversimplification because substantial heterogeneity in cardiovascular risk can exist among individuals with diabetes, based on age, duration of diabetes and other comorbidities [4, 8]. This supports the argument for more nuanced approaches (i.e. the second and third approaches above; Text box) to estimate absolute risk of cardiovascular disease in individuals with diabetes using multivariable prediction models that account for multiple cardiovascular disease risk factors.

A challenge is posed by the limitations of the prediction models currently used for assessing cardiovascular risk in people with diabetes. In 2012, van Dieren et al observed that of the 45 models used for predicting cardiovascular disease in individuals with diabetes reported in the literature, 33 were developed in the general population (i.e. the second approach above; Text box) and 12 were specifically developed using diabetic populations (i.e. the third approach above; Text box) [9]. The most commonly included predictors were clinical characteristics (age, sex, tobacco use) and lipid biomarkers (e.g. total cholesterol, LDL). Although many predictive models exist, only a minority have been externally validated in new diabetic populations and tested for predictive accuracy. Even the models that have been externally validated, such as the Framingham risk score [10] and the United Kingdom Prospective Diabetes Study (UKPDS) model [11], have limited ability to accurately estimate cardiovascular risk in type 2 diabetes $[12,13]$.

\section{Improving prediction of cardiovascular disease in individuals with diabetes}

Risk models for use in individuals with diabetes typically do not include information from biomarkers other than cholester$\mathrm{ol}$ and, occasionally, $\mathrm{HbA}_{1 \mathrm{c}}$ and albuminuria. This raises the question of whether adding novel biomarkers would improve cardiovascular risk prediction in individuals with diabetes.

What are the strategies for determining whether a novel biomarker improves the ability to predict cardiovascular disease risk? Risk prediction can only be improved by a novel biomarker if it provides additional information that is not explained by established cardiovascular disease risk factors such as age, tobacco use and lipid levels. Documenting a statistically significant association between a novel biomarker and cardiovascular disease in models adjusting for conventional risk factors is an important first step. Nonetheless, showing an association is not sufficient to establish whether the biomarker improves risk prediction $[14,16]$. Several additional metrics exist to assess whether a novel biomarker improves risk prediction: discrimination, calibration, and reclassification.

Discrimination addresses the model's ability to distinguish between individuals who will develop the outcome and those who will not. The most commonly used discrimination measure is the area under the receiver operating characteristic curve, also known as the $\mathrm{C}$-statistic. Values range from 0.5 (no discrimination ability) to 1.0 (perfect discrimination). Thus, a possible approach to demonstrating the utility of a novel biomarker is to evaluate whether the $\mathrm{C}$-statistic 
increases when the biomarker is added to conventional risk factors [14].

Calibration measures, such as the Hosmer-Lemeshow statistic, address the agreement between the risk predicted by the model compared with actual event rates in the population. Well-calibrated models yield accurate absolute risk estimates, which facilitate the assessment of the risks and benefits of various therapeutic approaches $[14,15]$. For instance, an intervention associated with a $20 \%$ reduction in cardiovascular disease risk but also with a high incidence of adverse effects might be more reasonable to consider in individuals at high absolute risk of cardiovascular disease.

Reclassification addresses the question of whether the addition of a biomarker leads to an appropriate change in the risk categorisation of individuals across a risk threshold. The most widely used measure of reclassification is the net reclassification improvement (NRI). An appropriate reclassification is one in which individuals who ultimately experience an event are reclassified into a higher risk group and individuals who do not experience an event are reclassified into a lower risk group [14]. The NRI is most useful when clearly defined risk thresholds exist for a condition and when these are tied to treatment decisions.

Because the pathogenesis of cardiovascular disease is multifactorial, no single biomarker can differentiate between individuals with diabetes who will and will not develop cardiovascular disease. Thus, clinically significant improvements in discrimination and reclassification in risk prediction models (second and third approaches; Text box) are unlikely to occur without the addition of multiple biomarkers. Using simulation, we have previously assessed how many hypothetical biomarkers might be required to produce large increases in the C-statistic (Fig. 1) [14]. The answer depends on the average correlation between pairs of biomarkers. Fewer biomarkers are required when the average correlation between biomarkers is low.

\section{Examples of cardiovascular disease biomarkers in individuals with diabetes}

Most investigations of cardiovascular disease biomarkers in diabetes have been hypothesis-driven, candidate biomarker studies focusing on known pathophysiological pathways, including those related to cardiac stress (N-terminal prohormone of b-type natriuretic peptide [NTproBNP] [8, 17-23] and high-sensitivity troponin $\mathrm{T}$ [hsTnT] [8, 17, 24]), inflammation (high-sensitivity Creactive protein [hsCRP] [8, 25], IL-6 [17], IL-15 [17]), matrix remodelling (matrix metalloproteinases and osteopontin [17, 23]), lipids [26], endothelial dysfunction (albuminuria [27]) and diabetes pathophysiology (AGEs) [17, $28]$ ). As in the general population, there is good evidence

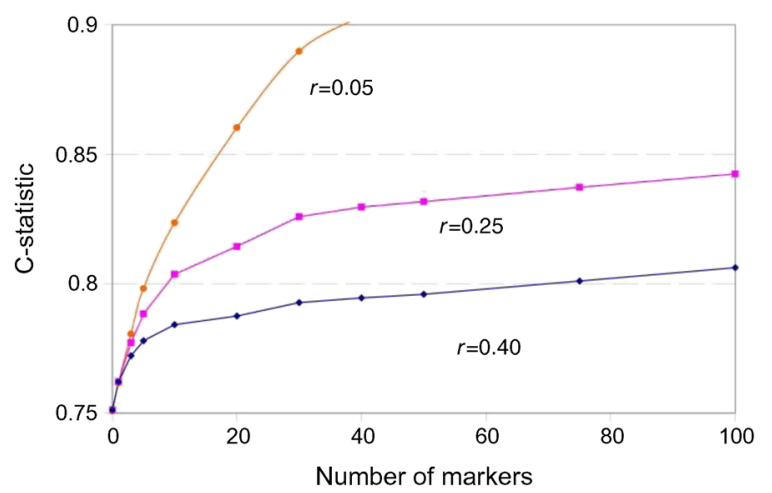

Fig. 1 Increases in discrimination achieved by adding simulated biomarkers, according to the degree of correlation between biomarkers. The simulated HR for the outcome is 1.35 per SD increment in the biomarker. The $y$-axis displays the $\mathrm{C}$-statistic from a model containing traditional risk factors plus a varying number of simulated biomarkers ( $x$-axis), each with a fixed association with the outcome. Each of the three curves represents a different degree of biomarker-biomarker correlation. The simulation was performed by M. Pencina, Boston University, (c) 2011 Wolters Kluwer Health Inc. Figure adapted with permission from Wang (2011) (http:// circ.ahajournals.org/content/123/5/551.long) [14]

in diabetic populations that NT-proBNP [8, 17-23], hsTnT $[8,17,24]$ and hsCRP $[8,25]$ are predictive of cardiovascular disease.

There have been numerous publications on the associations of single biomarkers with cardiovascular risk in diabetes but simultaneous evaluations of large numbers of biomarkers in diabetic populations have been limited. Table 1 presents selected studies in diabetic cohorts that evaluated at least 20 cardiovascular disease biomarkers simultaneously and reported changes in cardiovascular disease prediction beyond traditional risk factors [17, 19, 23]. The SUMMIT consortium [17] evaluated 42 candidate biomarkers, from a variety of physiological pathways previously reported to be associated with cardiovascular disease, in individuals with type 2 diabetes. The consortium identified six biomarkers whose combination led to moderate improvements in cardiovascular disease prediction, in terms of both discrimination and reclassification, beyond clinical covariates: NT-proBNP, hsTnT, IL-6, IL15, apolipoprotein C-III and soluble receptor for AGE. In another study, van der Leeuw et al [23] (Table 1, Fig. 2) evaluated 23 candidate biomarkers in two cohorts of individuals with type 2 diabetes and found that NT-proBNP, osteopontin, and matrix metalloproteinase 3 , on an individual basis as well as in combination, improved the prediction of cardiovascular events beyond traditional risk factors. Gerstein et al [19] (Table 1) evaluated 237 biomarkers in 8401 individuals with dysglycaemia (type 2 diabetes, impaired fasting glucose or impaired glucose tolerance) and identified ten biomarkers whose combination improved the prediction of a composite cardiovascular outcome (myocardial infarction, stroke or 


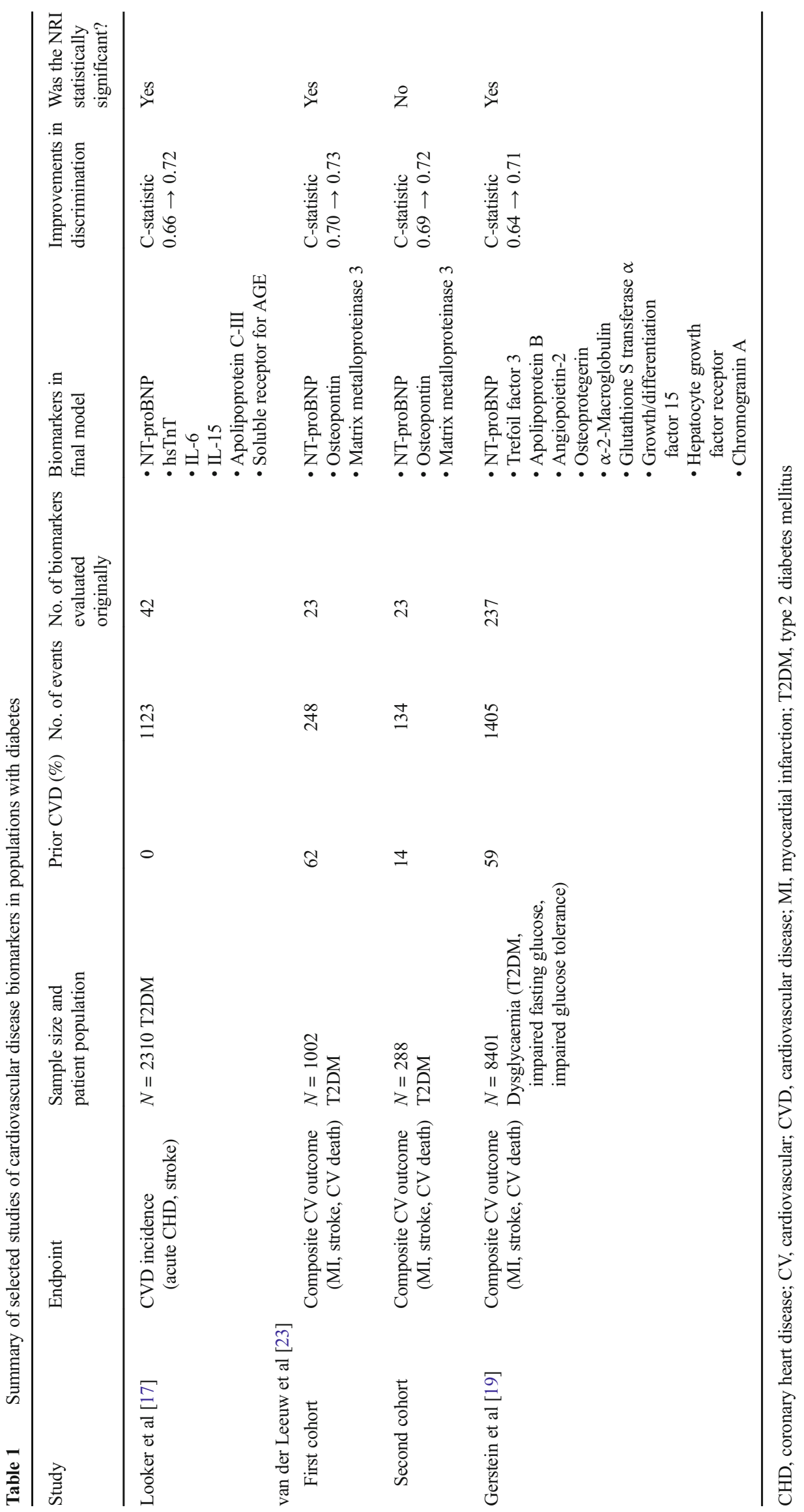




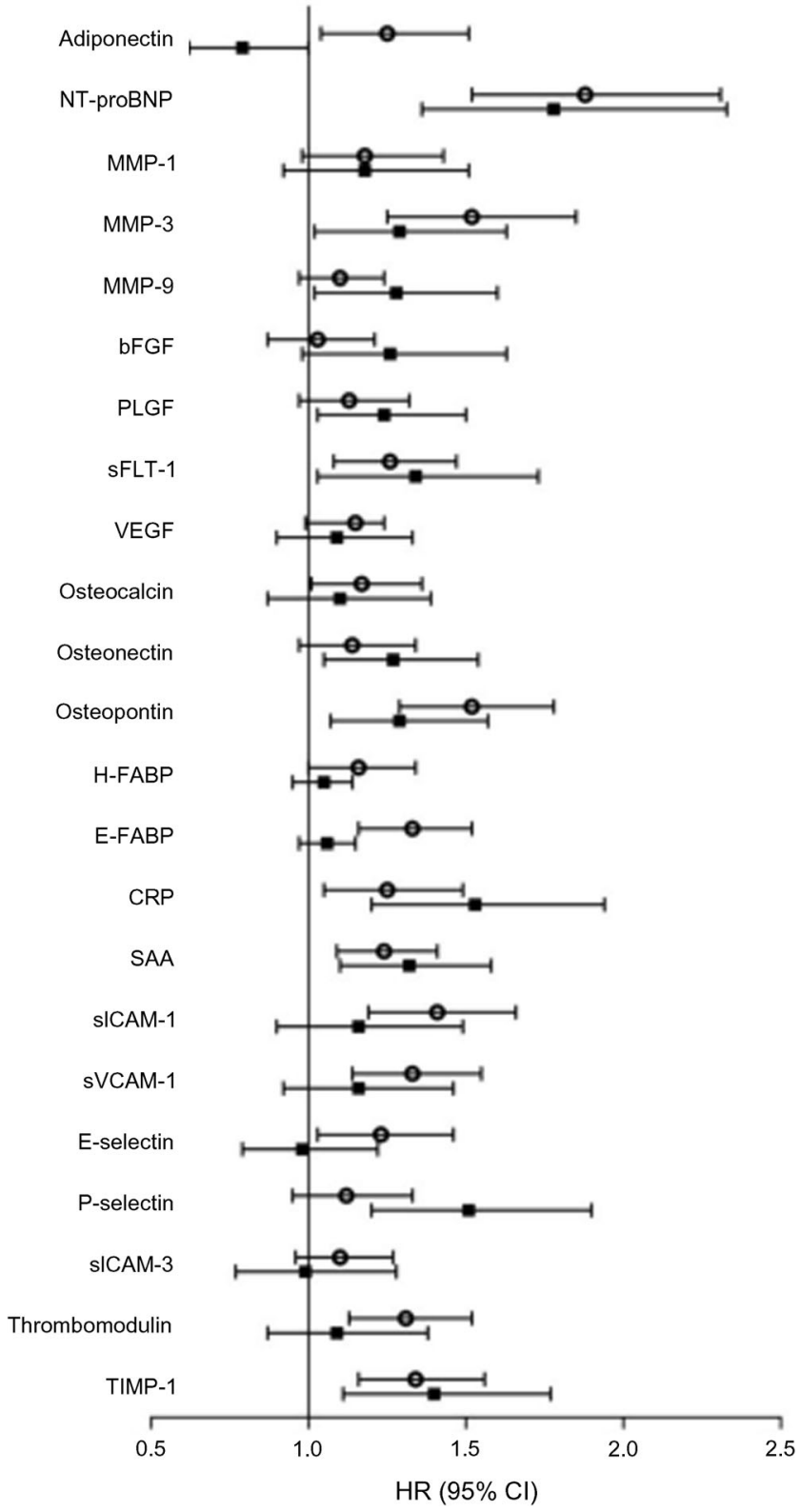

Fig. 2 Multivariable adjusted HRs for risk of major cardiovascular events for the highest vs the lowest quartile of each candidate biomarker in two type 2 diabetes cohorts: white circles, Second Manifestations of Arterial Disease (SMART) study; black squares, European Prospective Investigation into Cancer and Nutrition-Netherlands (EPIC-NL). Analyses were adjusted for sex, age at diabetes diagnosis, duration of diabetes, $\mathrm{HbA}_{1 \mathrm{c}}$, systolic blood pressure, total cholesterol/HDL ratio, urinary albumin/creatinine ratio, smoking status and previous cardiovascular event. Figure reproduced with minor adaptations from van der Leeuw et al [23], (C) 2016 under the Creative Commons Attribution 4.0 International (CC BY) license (https://creativecommons.org/licenses/by/ 4.0/legalcode). bFGF, basic fibroblast growth factor; CRP, C-reactive protein; E-FABP, epidermal-type fatty acid binding protein; H-FABP, heart-type fatty acid binding protein; MMP, matrix metalloproteinase; PLGF, placental growth factor; SAA, serum amyloid A; sFLT, soluble FMS-like tyrosine kinase; sICAM, soluble intercellular adhesion molecule; sVCAM, soluble vascular cell adhesion molecule; TIMP, tissue inhibitor of matrix metalloproteinase; VEGF, vascular endothelial growth factor cardiovascular death) beyond clinical covariates, with an improvement in the C-statistic from 0.64 to 0.71 .

These data suggest that certain biomarkers and biomarker combinations can lead to modest improvements in risk discrimination, beyond traditional cardiovascular risk factors. Estimates of NRI vary, though such estimates can depend on the selection of risk thresholds which do not always conform to clinical guidelines. 'Category-free' NRI measures have been developed that do not depend on the selection of thresholds, although such measures may provide less insight regarding the clinical utility of biomarker data. These observations lead to the question of whether novel biomarker information will have a clinical impact (discussed below).

\section{Applications of cardiovascular disease risk prediction models in clinical practice}

As already discussed, risk prediction models (the second and third approaches above; Text box) have been developed to estimate absolute risk of cardiovascular disease in individuals with diabetes. Risk prediction models have a clinical impact only if they are adopted into clinical practice and if their use in the clinical setting changes providers' management decisions (with regard to therapy, screening and education) and/or patients' behaviour [29-31]. To what extent are cardiovascular disease risk prediction models incorporated into current clinical guidelines issued by major medical societies? The European Society of Cardiology (ESC) and EASD 2013 guidelines [32] mention several available cardiovascular disease risk calculators for individuals with diabetes and also mention several cardiovascular disease biomarkers in diabetes. However, they do not explicitly advocate the use of risk calculators or biomarkers to guide clinical management. The ESC/EASD 2013 guidelines recommend classifying all individuals with diabetes as being at 'high risk' of cardiovascular disease (based solely on the presence of diabetes, without any other cardiovascular risk factor or evidence of target organ damage) or at 'very high risk' (documented cardiovascular disease, severe chronic kidney disease or presence of at least one cardiovascular risk factor or target organ damage). Thus, these guidelines recommend statin therapy for most individuals with diabetes.

The American Heart Association (AHA)/American College of Cardiology (ACC) 2013 guidelines recommend statin therapy for all individuals with diabetes aged 40 75 years, based solely on the presence of diabetes, and recommend that statin therapy be considered for patients with diabetes outside that age range based on the individuals' potential for cardiovascular disease risk reduction and personal preferences [33]. The AHA/ACC 2013 guidelines encourage healthcare providers to calculate the absolute risk using the 
AHA/ACC Pooled Cohort Risk Assessment Equation to guide the intensity of statin treatment in diabetic individuals who are 40-75 years of age.

In summary, the current lipid management guidelines from major European and American medical societies recommend statin therapy for most individuals with diabetes, based solely on the presence of diabetes, reflecting the first approach discussed above (Text box) which considers diabetes as a cardiovascular disease risk equivalent. However, even with this approach, risk prediction models and biomarker data may be used to inform the intensity of statin treatment or to help communicate risk to patients.

It is worth repeating that substantial heterogeneity in the risk of cardiovascular disease exists among individuals with diabetes $[4,8]$. This raises the question whether a more nuanced approach to the treatment of cardiovascular risk in individuals with diabetes should be considered. In one study, individuals with type 2 diabetes and multiple risk factors for cardiovascular disease had a 2 year rate of cardiovascular death, myocardial infarction or ischaemic stroke of $20 \%$ when levels of NT-proBNP, hsTnT and hsCRP were elevated. In contrast, the risk was only $1.6 \%$ for those with no elevation in any of these three biomarkers [8]. The absolute risk would likely be even lower in younger individuals with no clinical risk factors other than diabetes. According to both ESC/EASD and AHA/ACC guidelines, a 40-year-old individual with diabetes without any other clinical risk factors should be given statin therapy. However, if biomarker data revealed that this individual had an extremely low 10 year risk of cardiovascular events, it may be reasonable to defer statin therapy.

Thus, it remains possible, though unproven, that refinements in risk prediction may inform the application of current clinical guidelines, the aggressiveness of lipid reduction strategies, use of adjunctive medications beyond statins and communication of risk to patients. Very few publications have reported the impact of risk models on clinical management, not to mention clinical outcomes [9]. Well-designed, prospective studies examining the effectiveness of different screening and prevention strategies can be expensive and take many years to complete [29].

\section{Cardiovascular risk and management in individuals with prediabetes}

In contrast to diabetes, other states of hyperglycemia such as prediabetes (including impaired fasting glucose and impaired glucose tolerance) are not currently considered to be cardiovascular disease risk equivalents. Cardiovascular risk is elevated in individuals with prediabetes and hyperglycaemic conditions other than diabetes [34] but risk models such as the Framingham risk score and the AHA/ACC risk calculator do not account for these conditions. Moreover, there are no specific recommendations regarding the management of cardiovascular risk in individuals with prediabetes. Individuals with prediabetes are advised to institute healthy lifestyle changes (diet and exercise) but otherwise are treated the same as nondiabetic individuals regarding cardiovascular risk management. For instance, neither the ESC/EASD nor the AHA/ ACC guidelines for lipid management currently distinguish between individuals with pre-diabetes and those without diabetes.

Nonetheless, diabetes and prediabetes are not binary outcomes but rather fall along a continuum. Individuals with advanced prediabetes and those with early, mild diabetes may have a similar risk of cardiovascular disease. Thus, using the clinical onset of diabetes as the starting point for cardiovascular disease risk management may be too late. If biomarkers could refine the prediction of risk in prediabetic individuals, then those who would benefit from more aggressive risk management might be identified. An additional consideration is preventing diabetes itself since strategies aimed at preventing diabetes also lower cardiovascular disease risk. Identifying those at highest risk of developing diabetes would help target diabetes prevention efforts. Most of the currently known biomarkers offer only a modest improvement in risk prediction for type 2 diabetes beyond traditional risk factors [35]. However, recent technologies enabling high-throughput profiling of metabolic status (metabolomics) have identified novel biomarkers that improve prediction of diabetes and show promise for the future [36].

\section{Biomarkers as an approach to increasing the efficiency of clinical trials}

The US Food and Drug Administration requires that manufacturers of novel diabetes medications conduct large-scale outcomes trials to evaluate the cardiovascular risks of these drugs. Despite the elevated risk of cardiovascular disease in individuals with diabetes, short-term event rates are still moderate, meaning that very large sample sizes are required for adequately powered clinical trials. Accordingly, recent and ongoing cardiovascular trials of diabetes medications have had sample sizes of 3300-17,000 individuals [37]. Biomarkers that improve the identification of high-risk individuals with diabetes may allow smaller trials to be performed in these selected subgroups.

\section{Future directions}

As noted above, diabetes is a heterogeneous disease. The physiology of diabetes may differ by sex, race and age. Thus, it is conceivable that the predictive ability of cardiovascular disease biomarkers in individuals with diabetes may 
differ by clinical covariates. In non-diabetic individuals, biomarkers may perform differently according to clinical characteristics (e.g. in healthy postmenopausal women, hsCRP is an even stronger predictor of cardiovascular disease than is LDL [38]). There are few analogous data in diabetic populations but investigations to determine whether clinical characteristics such as sex, race and age influence the association of cardiovascular disease biomarkers with events are warranted. This information may be particularly useful in subgroups of younger individuals in whom the average absolute risk of events is low despite the presence of diabetes.

A related question is whether certain biomarkers may improve cardiovascular disease risk prediction selectively in diabetic vs non-diabetic individuals. Because the distribution of certain biomarkers may differ by diabetes status, their abilities to predict cardiovascular disease may also differ by diabetes status [39]. This appears to be the case for glucose levels and for markers of glucose-mediated damage. For example, whereas higher glucose levels are positively associated with cardiovascular disease risk in both diabetic and non-diabetic individuals $[40,41], \mathrm{HbA}_{1 \mathrm{c}}$ levels improve cardiovascular disease risk prediction in individuals with diabetes $[42,43]$ but not consistently in the general population $[44,45]$. In addition, markers of glucose-mediated damage, such as urinary microalbumin and AGEs, may be particularly useful for predicting cardiovascular disease risk in individuals with diabetes $[17,43]$. In contrast, the predictive abilities of C-reactive protein [46], fibrinogen [47] or $\gamma$-glutamyltransferase [48] do not appear to differ by diabetes status. Whether the predictive abilities of many cardiovascular disease biomarkers are modified by diabetes status is largely unknown. Addressing these questions is valuable not only for improving risk prediction but also for understanding how the pathophysiology of cardiovascular disease differs in individuals with and without diabetes.

Another important area of future research is the discovery of new biomarkers of cardiovascular disease risk in individuals with diabetes. As discussed above, if a biomarker has a relatively low correlation with existing predictors, it is better able to improve prediction of disease (Fig. 1). Because most studies so far have focused on traditional pathophysiological pathways for cardiovascular disease, such as inflammation, cardiac injury and oxidative stress, the potential improvement in prediction afforded by identifying additional biomarkers from the same pathways may be limited as such biomarkers are likely to be correlated.

In contrast to candidate-based designs, hypothesis-free approaches such as proteomics and metabolomics can simultaneously evaluate hundreds or thousands of biomarkers and offer the potential of identifying biomarkers outside the traditionally studied pathophysiological pathways. Proteomicsand metabolomics-based studies in related fields have enabled the discovery of novel biomarkers that improve the prediction of new-onset type 2 diabetes [36] and incident renal disease in type 2 diabetes [49]. These studies highlight the potential value of analogous studies to identify biomarkers of cardiovascular disease among individuals with diabetes.

\section{Conclusion}

Treatment guidelines for the primary and secondary prevention of cardiovascular disease in diabetic populations do not fully account for the heterogeneity in risk among individuals with diabetes. Certain biomarkers and combinations of biomarkers can lead to moderate improvements in cardiovascular disease risk prediction in diabetes. A critical question is whether the resultant improvement in risk stratification would lead to changes in clinical care for individuals with diabetes. The prospects for biomarker-guided management in diabetes would be further enhanced by expanding the number of biomarkers that contribute to risk stratification. Efforts to identify these biomarkers should be aided by recent advances in platforms for biomarker discovery that enable investigations involving broad panels of biomarkers not restricted to selected pathways. Ultimately, evidence from randomised trials is needed to establish whether adding biomarkers to conventional clinical assessment could lead to improved patient outcomes.

Funding Work in the authors' laboratories is supported by the National Institutes of Health (R01 DK081572, R01 DK108159) and by the Vanderbilt University Medical Center Faculty Research Scholars Program.

Duality of interest The authors declare that there is no duality of interest associated with this manuscript.

Contribution statement Both authors were responsible for drafting the article and revising it critically for important intellectual content and both approved the final version.

\section{References}

1. The Emerging Risk Factors Collaboration (2010) Diabetes mellitus, fasting blood glucose concentration, and risk of vascular disease: a collaborative meta-analysis of 102 prospective studies. Lancet 375 : 2215-2222

2. Kannel WB, McGee DL (1979) Diabetes and glucose tolerance as risk factors for cardiovascular disease: the Framingham study. Diabetes Care 2:120-126

3. Haffner SM, Lehto S, Ronnemaa T, Pyorala K, Laakso M (1998) Mortality from coronary heart disease in subjects with type 2 diabetes and in nondiabetic subjects with and without prior myocardial infarction. N Engl J Med 339:229-234

4. Scirica BM (2017) Use of biomarkers in predicting the onset, monitoring the progression, and risk stratification for patients with type 2 diabetes mellitus. Clin Chem 63:186-195 
5. St Clair L, Ballantyne CM (2007) Biological surrogates for enhancing cardiovascular risk prediction in type 2 diabetes mellitus. Am J Cardiol 99:80B-88B

6. Berezin AE (2016) Cardiac biomarkers in diabetes mellitus: new dawn for risk stratification? Diabetes Metab Syndr https://doi.org/ 10.1016/j.dsx.2016.12.032

7. Echouffo-Tcheugui JB, Kengne AP (2013) On the importance of global cardiovascular risk assessment in people with type 2 diabetes. Prim Care Diabetes 7:95-102

8. Scirica BM, Bhatt DL, Braunwald E et al (2016) Prognostic implications of biomarker assessments in patients with type 2 diabetes at high cardiovascular risk: a secondary analysis of a randomized clinical trial. JAMA Cardiol 1:989-998

9. van Dieren S, Beulens JW, Kengne AP et al (2012) Prediction models for the risk of cardiovascular disease in patients with type 2 diabetes: a systematic review. Heart 98:360-369

10. D'Agostino RB Sr, Vasan RS, Pencina MJ et al (2008) General cardiovascular risk profile for use in primary care: the Framingham heart study. Circulation 117:743-753

11. Stevens RJ, Kothari V, Adler AI, Stratton IM, United Kingdom Prospective Diabetes Study Group (2001) The UKPDS risk engine: a model for the risk of coronary heart disease in type II diabetes (UKPDS 56). Clin Sci (Lond) 101:671-679

12. Kengne AP, Patel A, Colagiuri S et al (2010) The Framingham and UK prospective diabetes study (UKPDS) risk equations do not reliably estimate the probability of cardiovascular events in a large ethnically diverse sample of patients with diabetes: the action in diabetes and vascular disease: Preterax and Diamicron-MR controlled evaluation (ADVANCE) study. Diabetologia 53:821-831

13. Davis WA, Colagiuri S, Davis TM (2009) Comparison of the Framingham and United Kingdom prospective diabetes study cardiovascular risk equations in Australian patients with type 2 diabetes from the Fremantle diabetes study. Med J Aust 190:180-184

14. Wang TJ (2011) Assessing the role of circulating, genetic, and imaging biomarkers in cardiovascular risk prediction. Circulation 123:551-565

15. Cook NR (2007) Use and misuse of the receiver operating characteristic curve in risk prediction. Circulation 115:928-935

16. Ware JH (2006) The limitations of risk factors as prognostic tools. N Engl J Med 355:2615-2617

17. Looker HC, Colombo M, Agakov F et al (2015) Protein biomarkers for the prediction of cardiovascular disease in type 2 diabetes. Diabetologia 58:1363-1371

18. Gaede P, Hildebrandt P, Hess G, Parving HH, Pedersen O (2005) Plasma N-terminal pro-brain natriuretic peptide as a major risk marker for cardiovascular disease in patients with type 2 diabetes and microalbuminuria. Diabetologia 48:156-163

19. Gerstein HC, Pare G, McQueen MJ et al (2015) Identifying novel biomarkers for cardiovascular events or death in people with dysglycemia. Circulation 132:2297-2304

20. Hillis GS, Welsh P, Chalmers J et al (2014) The relative and combined ability of high-sensitivity cardiac troponin $\mathrm{T}$ and $\mathrm{N}$-terminal pro-B-type natriuretic peptide to predict cardiovascular events and death in patients with type 2 diabetes. Diabetes Care 37:295-303

21. Tarnow L, Gall MA, Hansen BV, Hovind P, Parving HH (2006) Plasma N-terminal pro-B-type natriuretic peptide and mortality in type 2 diabetes. Diabetologia 49:2256-2262

22. Bruno G, Landi A, Barutta F et al (2013) N-terminal probrain natriuretic peptide is a stronger predictor of cardiovascular mortality than C-reactive protein and albumin excretion rate in elderly patients with type 2 diabetes: the Casale Monferrato population-based study. Diabetes Care 36:2677-2682

23. van der Leeuw J, Beulens JW, van Dieren S et al (2016) Novel biomarkers to improve the prediction of cardiovascular event risk in type 2 diabetes mellitus. J Am Heart Assoc 5 https://doi.org/10. 1161/JAHA. 115.003048
24. Hendriks SH, van Dijk PR, van Hateren KJ et al (2016) Highsensitive troponin $\mathrm{T}$ is associated with all-cause and cardiovascular mortality in stable outpatients with type 2 diabetes (ZODIAC-37). Am Heart J 174:43-50

25. Landman GW, Kleefstra N, Groenier KH et al (2016) Inflammation biomarkers and mortality prediction in patients with type 2 diabetes (ZODIAC-27). Atherosclerosis 250:46-51

26. Alshehry ZH, Mundra PA, Barlow CK et al (2016) Plasma lipidomic profiles improve on traditional risk factors for the prediction of cardiovascular events in type 2 diabetes mellitus. Circulation 134:1637-1650

27. Gerstein HC, Mann JF, Yi Q et al (2001) Albuminuria and risk of cardiovascular events, death, and heart failure in diabetic and nondiabetic individuals. JAMA 286:421-426

28. Meerwaldt R, Graaff R, Oomen PH et al (2004) Simple noninvasive assessment of advanced glycation end product accumulation. Diabetologia 47:1324-1330

29. Moons KG, Kengne AP, Grobbee DE et al (2012) Risk prediction models: II. external validation, model updating, and impact assessment. Heart 98:691-698

30. Moons KG, Altman DG, Vergouwe Y, Royston P (2009) Prognosis and prognostic research: application and impact of prognostic models in clinical practice. BMJ 338:b606

31. Reilly BM, Evans AT (2006) Translating clinical research into clinical practice: impact of using prediction rules to make decisions. Ann Intern Med 144:201-209

32. Authors/Task Force Members, Ryden L, Grant PJ et al (2013) ESC guidelines on diabetes, pre-diabetes, and cardiovascular diseases developed in collaboration with the EASD: the task force on diabetes, pre-diabetes, and cardiovascular diseases of the European Society of Cardiology (ESC) and developed in collaboration with the European Association for the Study of Diabetes (EASD). Eur Heart J 34:3035-3087

33. Stone NJ, Robinson JG, Lichtenstein AH et al (2014) 2013 ACC/ AHA guideline on the treatment of blood cholesterol to reduce atherosclerotic cardiovascular risk in adults: a report of the American College of Cardiology/American Heart Association task force on practice guidelines. Circulation 129:S1-S45

34. Anand SS, Dagenais GR, Mohan V et al (2012) Glucose levels are associated with cardiovascular disease and death in an international cohort of normal glycaemic and dysglycaemic men and women: the EpiDREAM cohort study. Eur J Prev Cardiol 19:755-764

35. Echouffo-Tcheugui JB, Dieffenbach SD, Kengne AP (2013) Added value of novel circulating and genetic biomarkers in type 2 diabetes prediction: a systematic review. Diabetes Res Clin Pract 101:255269

36. Wang TJ, Larson MG, Vasan RS et al (2011) Metabolite profiles and the risk of developing diabetes. Nat Med 17:448-453

37. Singh AK, Singh R (2017) SAVOR-TIMI to SUSTAIN-6: a critical comparison of cardiovascular outcome trials of antidiabetic drugs. Expert Rev Clin Pharmacol 10:429-442

38. Ridker PM, Hennekens CH, Buring JE, Rifai N (2000) C-reactive protein and other markers of inflammation in the prediction of cardiovascular disease in women. N Engl J Med 342:836-843

39. Echouffo-Tcheugui JB, Ogunniyi MO, Kengne AP (2011) Estimation of absolute cardiovascular risk in individuals with diabetes mellitus: rationale and approaches. ISRN Cardiol 2011: 242656

40. Coutinho M, Gerstein HC, Wang Y, Yusuf S (1999) The relationship between glucose and incident cardiovascular events. A metaregression analysis of published data from 20 studies of 95,783 individuals followed for 12.4 years. Diabetes Care 22:233-240

41. Selvin E, Marinopoulos S, Berkenblit G et al (2004) Meta-analysis: glycosylated hemoglobin and cardiovascular disease in diabetes mellitus. Ann Intern Med 141:421-431 
42. Chamnan P, Simmons RK, Sharp SJ, Griffin SJ, Wareham NJ (2009) Cardiovascular risk assessment scores for people with diabetes: a systematic review. Diabetologia 52:2001-2014

43. Kengne AP, Patel A, Marre M et al (2011) Contemporary model for cardiovascular risk prediction in people with type 2 diabetes. Eur $\mathrm{J}$ Cardiovasc Prev Rehabil 18:393-398

44. Simmons RK, Sharp S, Boekholdt SM et al (2008) Evaluation of the Framingham risk score in the European prospective investigation of Cancer-Norfolk cohort: does adding glycated hemoglobin improve the prediction of coronary heart disease events? Arch Intern Med 168:1209-1216

45. Selvin E, Coresh J, Golden SH, Brancati FL, Folsom AR, Steffes MW (2005) Glycemic control and coronary heart disease risk in persons with and without diabetes: the atherosclerosis risk in communities study. Arch Intern Med 165:1910-1916

46. Kengne AP, Batty GD, Hamer M, Stamatakis E, Czernichow S (2012) Association of C-reactive protein with cardiovascular disease mortality according to diabetes status: pooled analyses of 25,979 participants from four U.K. prospective cohort studies. Diabetes Care 35:396-403

47. Kengne AP, Czernichow S, Stamatakis E, Hamer M, Batty GD (2013) Fibrinogen and future cardiovascular disease in people with diabetes: aetiological associations and risk prediction using individual participant data from nine community-based prospective cohort studies. Diab Vasc Dis Res 10:143-151

48. Kengne AP, Czernichow S, Stamatakis E, Hamer M, Batty GD (2012) Gamma-glutamyltransferase and risk of cardiovascular disease mortality in people with and without diabetes: pooling of three British health surveys. J Hepatol 57:1083-1089

49. Pena MJ, Jankowski J, Heinze G et al (2015) Plasma proteomics classifiers improve risk prediction for renal disease in patients with hypertension or type 2 diabetes. J Hypertens 33:2123-2132 